\title{
Evaluación de una técnica de hibridación reversa para identificación rápida de micobacterias en Chile
}

TAMARA LEIVA C.*

Evaluation of a reverse hybridization assay for the identification of Mycobacterium in Chile

Objective: Identification for Mycobacterium assay based in the new technology of reverse hybridization DNA probe assay was evaluated (Line Probe Assays-LiPAs). Methods: 74 strains belonging to 23 mycobacterial species or complex classified previously by classical biochemical methods, genetic probes and PRA (patterns of restriction analysis), with and without specific pattern expected to be identified at specie level were analysed.The utilized test, GenoType CM (Hain Lifescience, Nehren, Alemania), is able of identifying 14 of the most common mycobacterial species after a multiplex PCR technique targeting a 23S rRNA gene region followed by reverse hybridization technology. Results: Sensitivity of $94.0 \%$ (95\% CI: 84.4-98.0\%) and specificity of 88.0\% (95\% CI:46.7-99.3\%) were obtained with the assay. Conclusion: GenoType CM is an appropriated tool for the identification of Mycobacteria, rapid, sensitive, operational in the current working conditions of the National Reference Laboratory of Mycobacteria in Chile and it might constitute a real breakthrough for shortening the time delay in the procedure, providing a better opportunity to use treatment only in cases where it is required.

Key words: Rapid mycobacterial identification, Line Probe Assays-LiPAs, GenoType CM, reverse hybridization DNA.

\section{Resumen}

Objetivos: Se evalúa una técnica para la identificación de micobacterias basada en la nueva tecnología de hibridación en tiras con sondas (Line Probe Assays-LiPAs). Métodos: Se analizaron 74 cepas, correspondientes a 23 especies y/o complejos, preclasificadas mediante pruebas bioquímicas tradicionales, sondas genéticas y PRA (análisis de patrones de restricción), identificables y no identificables a nivel de especie por el kit utilizado. El kit evaluado, GenoType CM (Hain Lifescience, Nehren, Alemania), permite la identificación genética molecular de 14 de las especies micobacterianas más comunes, mediante una PCR múltiple e hibridación reversa del producto en tiras con sondas de regiones genéticas de ARNr de 23S. Resultados: Con la utilización de este ensayo para identificación de Micobacterias se obtuvo 94,0\% (CI 95\% 84,4-98,0) de sensibilidad y 88,0\% (CI 95\% 46,7-99,3) de especificidad totales. Conclusiones: Se concluye que GenoType CM constituye una herramienta adecuada para la identificación de micobacterias, rápida, sensible, operativa en las actuales condiciones de trabajo del Laboratorio de Referencia Nacional de Micobacterias en Chile y que podría constituir un avance para el acortamiento en los tiempos que demora el proceso, lo que implica una mejor oportunidad de aplicación de tratamiento sólo en los casos en que éste sea requerido.

Palabras clave: Rápida identificación de micobacterias, Line Probe Assays-LiPAs, GenoType CM, hibridación reversa.

* Sección Micobacterias, Laboratorio Biomédico Nacional, Instituto de Salud Pública de Chile. 


\section{Introducción}

Las micobacterias están presentes en una gran variedad en la naturaleza y en diversos nichos ecológicos, existiendo hasta ahora más de 100 especies descritas. De éstas, algunas son saprófitas y otras se comportan como patógenas oportunistas. Pueden producir Micobacteriosis pulmonar, cutánea, abscesos, septicemias, entre otros cuadros clínicos, y afectar especialmente a pacientes inmunosuprimidos por $\mathrm{VIH}+/$ SIDA o trasplantes. La discriminación de las micobacterias ambientales saprófitas de las potencialmente patógenas es un desafío diagnóstico relevante para la decisión y aplicación correcta y oportuna de tratamiento al paciente ${ }^{1-3}$.

En Chile, en el último estudio de frecuencia de Micobacterias se detectó que el 91,3\% de las cepas aisladas correspondían a complejo Mycobacterium tuberculosis y 8,7\% a micobacterias medioambientales. Dentro de esta últimas, $M y$ cobacterium kansasii, Complejo Mycobacterium avium-intracellulare, Mycobacterium chelonae, Mycobacterium fortuitum y Mycobacterium gordonae fueron las más comúnmente encontradas (Scappaticcio y cols., datos no publicados).

La identificación de micobacterias actualmente se hace mediante pruebas bioquímicas tradicionales, basadas en características fenotípicas como morfología, tinción Ziehl Neelsen, velocidad de crecimiento, temperatura óptima de crecimiento, producción de pigmento, metabolismo enzimático y sensibilidad a drogas antituberculosas, sondas genéticas y análisis de patrones de restricción (PRA) $)^{1,4}$ Sin embargo, cada una de éstas representan desventajas como son requerir semanas y hasta meses para su realización y no siempre permitir identificación a nivel de especie en el caso de las pruebas fenotípicas, estar limitadas a un reducido número de especies, en el caso de las sondas comerciales, o requerir de gran experiencia para una correcta identificación de bandas, en el caso de PRA.

Se requieren metodologías con mayor rapidez y efectividad en la identificación de especies micobacterianas para la correcta y oportuna aplicación del tratamiento.

Las relativamente nuevas técnicas basadas en la secuencia de ADN para identificación de micobacterias que se han desarrollado en los últimos 15 años, como secuenciación, RFLP, PCR en tiempo real, entre otras, requieren equipamientos muy sofisticados y/o de gran experiencia ${ }^{5,6}$.

Recientemente se han desarrollado técnicas con la tecnología de hibridación reversa de un producto de PCR de una secuencia genética de
ARNr de 23S llamados LiPAs (line probe assays). Son técnicas estandarizadas, validadas en la Comunidad Europea y de rápida respuesta, que no requieren costosos equipamientos o gran experiencia. Una de ellas permite la identificación simultánea, a nivel de especie, de las micobacterias más relevantes desde punto de vista clínico, a partir de muestras clínicas, cultivos líquidos o sólidos, y otras identifican complejo Mycobacterium tuberculosis y detectan genes de resistencia a drogas de primera y segunda línea ${ }^{5,7-14}$.

El objetivo de este trabajo es evaluar una de estas técnicas de hibridación reversa, GenoType CM (Hain Lifescience, Nehren, Alemania) en la identificación de micobacterias provenientes de cepas de pacientes en Chile y en la factibilidad de su implementación en el trabajo de rutina del Laboratorio Nacional de Referencia de Micobacterias del Instituto de Salud Pública.

\section{Materiales y Métodos}

\section{Cepas analizadas}

Se incluye un total de 74 cepas conformadas de la siguiente manera: 63 cepas derivadas de muestras de pacientes provenientes de la red de los Laboratorios de Bacteriología de la tuberculosis del país al Laboratorio de Referencia Nacional de Micobacterias (LRN) del Instituto de Salud Pública de Chile, 2 cepas aisladas de muestras de cerdos más una cepa ATCC (American type culture collection, USA), todas almacenadas en el cepario del LRN entre 2006 y 2010. Las cepas fueron seleccionadas, incluidas y analizadas con el siguiente criterio: al menos 12 especies y/o complejos de las especies detectadas por la técnica con GenoType CM (a nivel de especie única o asociada) y 9 que son no detectables por el ensayo a nivel de especie. Además, se incluyeron 8 cepas no micobacterianas, pero cercanamente relacionadas a éstas del género Actinomycetes, facilitadas por el laboratorio de Agentes emergentes y zoonóticos de la Sección de Bacteriología del Instituto de Salud Pública de Chile (Tabla 1).

\section{Caracterización de las cepas}

Las cepas incluidas en el estudio fueron preclasificadas por pruebas fenotípicas y/o genotípicas. La identificación fenotípica se realizó mediante el uso de la clasificación de Runyon (1959) en medios sólidos, por pruebas de identificación de rutina de micobacterias. Estas incluyen incubaciones a diversas temperaturas $(25,37 \mathrm{y}$ $42^{\circ} \mathrm{C}$ ), lectura precoz de subcultivos para detectar 
eventuales micobacterias de crecimiento rápido y la capacidad de formar pigmentos (cepas escotocromógenas, fotocromógenas y acromógenas). Se incluyen también pruebas de identificación bioquímicas como: niacina, nitrato, catalasa a temperatura ambiente y termoinactivada a $68^{\circ} \mathrm{C}$, ureasa, tween 80, cloruro de sodio, pirazinamidasa, susceptibilidad a cicloserina y TCH. Genotípicamente, fueron analizadas por sondas genéticas AccuProbe (GenProbe, San Diego, CA) de acuerdo a las indicaciones del fabricante y por análisis de patrones de restricción (PRA) según Araya y colegas ${ }^{4}$.

\section{Ensayo GenoType CM}

El ensayo de GenoType CM se realizó de acuerdo a las recomendaciones del fabricante. Brevemente, una asada de cultivo sólido de las micobacterias estudiadas fue extraída en 300 uL de agua destilada calidad biología molecular, y

Tabla 1. Distribución de cepas incluidas en el estudio

\begin{tabular}{|lc|}
\hline Especies/complejos de micobacterias & n de cepas \\
\hline Complejo M. TBC & 4 \\
\hline Complejo MAI & 12 \\
\hline Complejo terrae & 2 \\
\hline M. abscessus & 4 \\
\hline M. bovis & 2 \\
\hline M. bovis BCG & 1 \\
\hline M.chelonae & 3 \\
\hline M. chelonae-abscessus & 2 \\
\hline M. confluentis & 1 \\
\hline M. flavescens & 1 \\
\hline M. fortuitum & 4 \\
\hline M. gadium & 1 \\
\hline M. gastri & 1 \\
\hline M. gordonae & 1 \\
\hline M. kansasii & 6 \\
\hline M. mucogenicum & 5 \\
\hline M. peregrinum & 3 \\
\hline M. terrae & 1 \\
\hline M. tuberculosis & 4 \\
\hline M. nonchromogenicum & 3 \\
\hline M. smegmatis & 3 \\
\hline Nocardia & 1 \\
\hline Actinomyces no Nocardia & 1 \\
\hline Total & 1 \\
\hline
\end{tabular}

calentada a $95^{\circ} \mathrm{C}$ en baño termoregulado por 20 minutos, expuesta a baño de ultrasonido por 15 minutos, centrifugada a 13.000 g por 5 minutos y el ADN extraído fue traspasado a un nuevo tubo congelándose a $-70^{\circ} \mathrm{C}$. Para la amplificación, fueron usados $35 \mathrm{~mL}$ de una mezcla de nucleótidos primer (provistos por el kit), buffer de amplificación que contiene 2,5 mM de $\mathrm{MgCl}_{2}, 1,25 \mathrm{U}$ de Hot Start Taq polimerasa (Qiagen), $3 \mathrm{~mL}$ de agua desionizada calidad biología molecular y $5 \mathrm{~mL}$ de la suspensión inactivada en un volumen final de $45 \mathrm{~mL}$. El protocolo de amplificación consistió en 15 minutos de denaturación a $95^{\circ} \mathrm{C}$, seguido por 10 ciclos que comprenden $30 \mathrm{~s}$ a $95^{\circ} \mathrm{C}$ y $120 \mathrm{~s}$ a $58^{\circ} \mathrm{C}, 20$ ciclos que comprenden $25 \mathrm{~s}$ a $95^{\circ} \mathrm{C}, 40$ s a $53^{\circ} \mathrm{C}$, y $40 \mathrm{~s}$ a $70^{\circ} \mathrm{C}$ y una extensión final de 8 min a $70^{\circ} \mathrm{C}$. La hibridación y detección se desarrolló en un agitador con temperatura semiautomatizado, (Twincubator, Hain), que tiene incorporado el programa de agitaciones a diferentes temperaturas para cada etapa de la hibridación. Este comienza después de la denaturación de 20 $\mathrm{mL}$ producto de amplificación con un reactivo denaturante (contenido en el kit) durante 5 minutos en un pocillo plástico para 12 tiras. Se agrega $1 \mathrm{~mL}$ de tampón de hibridación precalentado a $40^{\circ} \mathrm{C}$, se mezcla y agita suavemente y se agrega una tira preidentificada por muestra en cada canal del pocillo plástico. La hibridación se realiza a $45^{\circ} \mathrm{C}$ por $0,5 \mathrm{~h}$, seguido por dos lavados. Para la detección colorimétrica de amplicones hibridizados, se agrega sucesivamente conjugado de estreptavidina con fosfatasa alcalina y sustrato, ambos diluídos 1/100 con buffer conjugado y buffer sustrato respectivamente. Después de un lavado final, las tiras se secan y se fijan en papel. Cada tira tiene 17 sondas, que incluyen 3 controles cada una: a) Control de conjugado, que evalúa la unión del conjugado a la sonda y reacción del sustrato, y debe aparecer para documentar la eficacia de esta reacción; b) Control universal, para detección de especies ricas en guanina citosina (todas las micobacterias y miembros del grupo de Gram positivas con alto contenido de Guanina+Citosina) y c) Control de género que documenta la presencia de un miembro del género Mycobacterium. En la lectura de bandas, sólo deben considerarse intensidades de coloración de bandas iguales o superiores al control universal. Cuando sólo el control de conjugado y control universal se tiñen como positivas pero el resto de bandas no aparece, se deben aplicar métodos adicionales para la identificación de la especie bacteriana correspondiente. La banda de control de género puede desaparecer a pesar de la presencia de ADN micobacteriano; sin embargo, siempre 


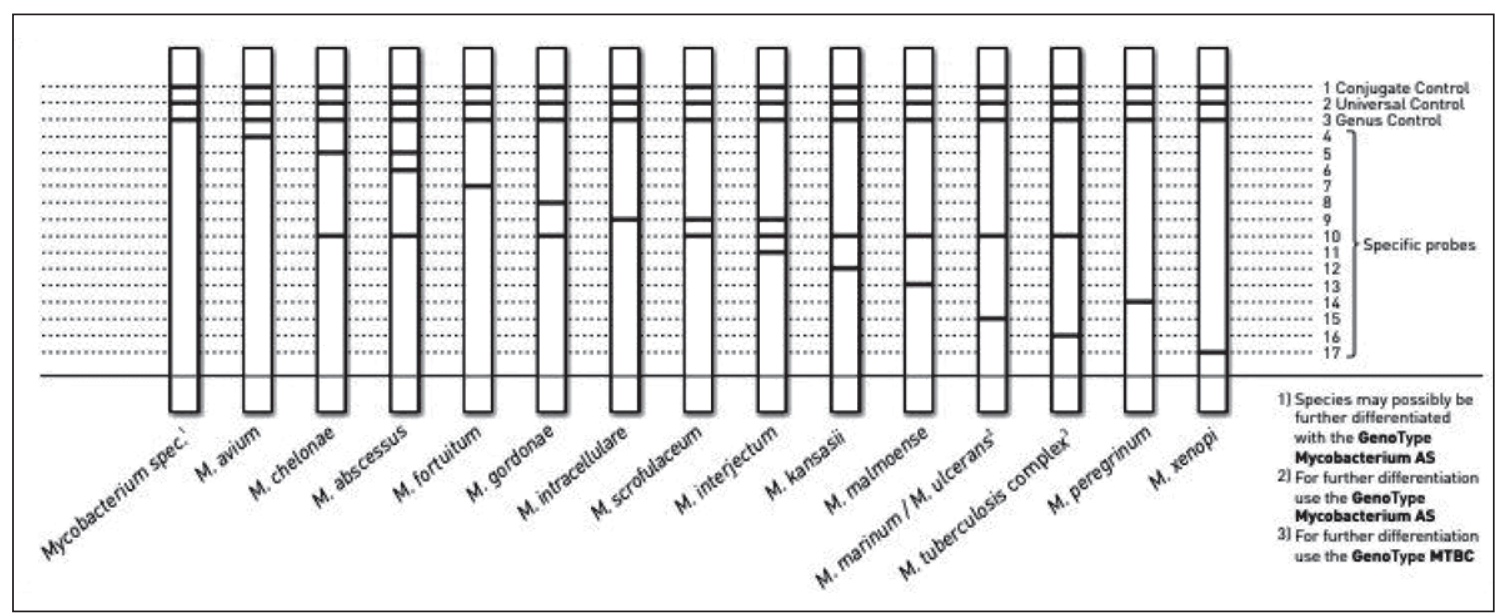

Figura 1. Cepas identificables por GenoType CM.

Tabla 2. Resultados de identificación de cepas incluidas en bandeos de GenoType CM

\begin{tabular}{|c|c|c|c|}
\hline $\begin{array}{l}\text { Resultados de GenoType CM } \\
\text { Identificables por la técnica }\end{array}$ & $\mathbf{n}^{*}$ & Patrón de bandeo & Identificación \\
\hline Complejo M. TBC & 4 & $1,2,3,10,16$ & Complejo $M$. TBC \\
\hline Complejo MAI & 10 & $1,2,3,4$ & M. avium ssp. \\
\hline Complejo MAI & 2 & $1,2,3,9$ & M. intracellulare \\
\hline M. abscessus & 3 & $1,2,3,5,6,10$ & M. abscessus \\
\hline M. abscessus & 1 & $1,2,3,10$ & Mycobacterium spec. \\
\hline M. bovis & 2 & $1,2,3,10,16$ & Complejo M. TBC \\
\hline M. bovis BCG & 1 & $1,2,3,10,16$ & Complejo M. TBC \\
\hline M. chelonae & 3 & $1,2,3,5,10$ & M. chelonae \\
\hline M. chelonae-abscessus & 2 & $1,2,3,5,6,10$ & M. abscessus \\
\hline M. fortuitum & 4 & $1,2,3,7,14$ & M. fortuitum \\
\hline M. gordonae & 6 & $1,2,3,8,10$ & M. gordonae \\
\hline M. kansasii & 5 & $1,2,3,10,12$ & M. kansasii \\
\hline M. peregrinum & 4 & $1,2,3,14$ & M. peregrinum \\
\hline M. tuberculosis & 5 & $1,2,3,10,16$ & Complejo M. TBC \\
\hline Total & 52 & & \\
\hline
\end{tabular}

*n = número de cepas.

que esté presente un patrón de bandeo específico de especie, la reacción de amplificación se lleva a cabo correctamente y el resultado del ensayo es válido.

Los patrones de bandeo y especies identificables por GenoType CM se muestran en la Figura 1. Permite la identificación de Complejo Mycobacterium tuberculosis y las 14 micobacterias ambientales más comúnmente aisladas y la lectura se realiza mediante el uso de una plantilla de bandeo de fácil interpretación.

\section{Resultados}

De las 74 cepas analizadas, 52 correspondieron a especies y/o complejos identificables a nivel de especie (única o asociada) por la técnica, mientras 14 correspondían a especies no identificables a nivel de especie, más 8 Actinomycetes como especies estrechamente relacionadas con el género Mycobacteria: 8 Nocardia y un Actinomyces.

El 100\% de los análisis tuvieron resultados interpretables. 
Tabla 3. Resultados de identificación de cepas no incluidas en bandeos de GenoType CM

\begin{tabular}{|lccl|}
\hline $\begin{array}{l}\text { Resultados de GenoType CM } \\
\text { No identificables }\end{array}$ & n* & Patrón de bandeo & Identificación \\
Complejo terrae & 2 & $1,2,3,10$ & Mycobacterium spec. \\
M. confluentis & 1 & $1,2,3$ & M. ssp \\
M. flavescens & 1 & $1,2,3$ & M. ssp \\
M. gadium tipo 1 & 1 & $1,2,3,5,10$ & M. chelonae \\
M. gastri & 1 & 1,2 & Gram positivo alto contenido G + C \\
M. mucogenicum & 3 & $1,2,3,10$ & Mycobacterium spec. \\
M. terrae & 3 & $1,2,3,10$ & Mycobacterium spec. \\
M. nonchromogenicum & 1 & $1,2,3,10$ & Mycobacterium spec. \\
M. smegmatis & 1 & $1,2,14$ & M. fortuitum \\
Nocardia & 7 & 1,2 & Gram positivo alto contenido G + C \\
\hline Actinomyces no Nocardia & 1 & $1,2,3,5,6,10$ & M. abscessus \\
\hline Total & 22 & & \\
\hline
\end{tabular}

*n = número de cepas. ${ }^{\#} \mathrm{G}+\mathrm{C}=$ Guanina + Citosina.

Tabla 4. Valores de sensibilidad y especificidad obtenidas por GenoType CM

\begin{tabular}{|lcc|}
\hline & \% sensibilidad & \% especificidad \\
\hline Sonda específica de género & $78,6(40,8-82,8)$ & $87,5(46,7-99,3)$ \\
\hline Sondas específicas de especie & $98,0(88,4-99,9)$ & $88,0(46,7-99,3)$ \\
\hline
\end{tabular}

De las 52 cepas potencialmente identificables a nivel de especie por la técnica, 51 (98\%) fueron correctamente identificadas (Tablas 2 y 4), mientras que de las 14 no identificables a nivel de especie, 11 (78,6\%) fueron correctamente detectadas como pertenecientes al género Mycobacterium (Tabla 4). De los 8 Actinomycetes, 7 fueron correctamente identificados como especie Gram positiva con alto contenido en Guanina+Citosina y uno fue mal identificado (Tabla 3).

De las cepas que debían ser identificadas a nivel de especie, una fue identificada sólo como Mycobacterium sin llegar a determinarse la especie (Mycobacterium abscessus fue identificada como Mycobacterium spec.).

De las cepas incluidas en el muestreo que no eran identificables a nivel de especie por GenoType CM, 2 fueron identificadas erróneamente (Mycobacterium gadium como Mycobacterium chelonae y Mycobacterium smegmatis como Mycobacterium fortuitum) y una micobacteria se identificó como Gram positivo con alto contenido en Guanina+Citosina sin llegar a ser identificada como micobacteria (Mycobacterium gastri). De los Actinomycetes estudiados, 7 Nocardia fueron correctamente identificadas como especie Gram positiva con alto contenido en Guanina+Citosina mientras un Actinomyces fue identificado como Mycobacterium abscessus. Con estos resultados se obtuvieron especificidades de 87,5 y $88 \%$ para la identificación de género y especie respectivamente.

\section{Discusión}

La evaluación de GenoType CM como nueva técnica para la identificación de micobacterias, dio como resultado un 94\% (IC 95\%: 84,4-98,0) de sensibilidad y un 88\% (IC 95\%: 46,7-99,3) de especificidad, lo que se acerca bastante a publicaciones internacionales de evaluaciones de la técnica ${ }^{5,8,10-13}$. A nuestro saber ésta es la primera evaluación y publicación que se realiza en la Región y en el país de GenoType CM.

Los resultados demuestran que es una técnica confiable para la identificación de cepas más comúnmente aisladas en Chile, aunque no el 100\% fue identificado a nivel de especie, por lo cual aspectos fenotípicos deben ser considerados para lograr la especificación en los casos en que el kit no identifique. Estos resultados se ven apoyados por resultados publicados previamente $e^{5,8,10,11}$. 
En el caso de identificación de cepas no contenidas para identificación en GenoType CM, 11 $(78,6 \%)$ fueron identificadas como pertenecientes al género de micobacterias; sin embargo, una de éstas fue identificada como Gram positiva con alto contenido en Guanina+Citosina, de lo que se concluye que no siempre un resultado de este tipo de bandeo descarta la presencia de micobacterias y es necesaria la observación de bacilos alcohol-ácido resistentes-BAAR de la colonia para confirmar o descartar la presencia de éstas. Este resultado también concuerda con publicaciones de investigadores europeos ${ }^{5}$. Las dos cepas mal identificadas, Mycobacterium gadium y Mycobacterium smegmatis, que fueron identificadas como Mycobacterium chelonae y Mycobacterium fortuitum respectivamente, fueron clasificadas por PRA, ya que no se dispone en nuestro laboratorio de pruebas bioquímicas que identifiquen a la primera como tal, ni que diferencien a las dos últimas. Sin embargo, la cepa recibida en el laboratorio que fue identificada por GenoType CM como Mycobacterium chelonae era cromógena lo que hace aceptar a Mycobacterium gadium y descartar Mycobacterium chelonae como identificación más probable. Por otro lado, entre Mycobacterium smegmatis y Mycobacterium fortuitum tampoco se dispone de pruebas bioquímicas discriminatorias definitivas y el PRA de ambas especies da bandas bastante parecidas.

En el caso de los Actinomyces, taxonómicamente cercanos a Micobacterias, organismos Gram positivos con alto contenido en Guanina+Citosina y que debían ser identificados como tales por la tira, uno de ellos fue mal identificado como Mycobacterium abscessus. Esto reafirma que deben ser considerados, por ejemplo, al comenzar el estudio, aspectos macroscópicos de la colonia, tinción alcohol ácido resistente del microorganismo y de esa manera detectar si se produjera alguna identificación incorrecta. En todo caso, de los Actinomyces, es Nocardia la que se encuentra más frecuentemente en las muestras recibidas en el laboratorio, cepa que fue correctamente identificada.

Si bien, recientemente se han publicado errores en resultados de la identificación de cepas mediante LiPAs, las especies involucradas no corresponden al patrón epidemiológico de micobacterias medioambientales de nuestro país ${ }^{13}$, (Scappaticcio A. y col., datos no publicados).

La implementación de la técnica fue totalmente operativa, ya que a nivel de LRN se cuenta con las dependencias necesarias para la realización de ésta: laboratorio BSL3 para la extracción de
ADN y tres áreas separadas para pre-PCR, PCR e hibridación.

Los resultados pueden ser obtenidos en 5 horas aproximadamente, por lo que es totalmente factible realizar la técnica y obtener resultados en una jornada de trabajo. Esto implica un gran avance respecto a los tiempos de demora actuales necesarios para la identificación de micobacterias mediante fenotipo, que pueden llegar a ser de semanas y hasta meses. Además mediante técnicas bioquímicas tradicionales no es siempre posible llegar a identificación a nivel de especie. La otra herramienta de la cual dispone el laboratorio de Micobacterias es la sonda, pero ésta presenta las limitaciones de identificar sólo Complejo $\mathrm{Myco-}$ bacterium tuberculosis, Complejo Mycobacterium avium, $M$. kansasii y Mycobacterium gordonae y tener un costo más alto que GenoType CM.

Se concluye que GenoType CM constituye una herramienta adecuada para la identificación de micobacterias, rápida, sensible, operativa en las actuales condiciones de trabajo del LRN de Micobacterias en Chile y que podría llegar a constituir un verdadero avance para el acortamiento en los tiempos de demora, lo que implica mayor oportunidad de aplicación de tratamiento sólo en los casos en que éste sea requerido.

\section{Agradecimientos}

Agradecemos a Rosario Lepe y Maritza Velasco por su "herencia" en Micobacterias; a Marcia Bahamondes por su apoyo en la preparación del texto; a Luis Rodríguez, por su apoyo en Bioestadística, a Hain Lifescience por el entrenamiento en la técnica y a Biomerieux por proveer los kits para el ensayo.

\section{Bibliografía}

1.- PRIMM T P, LUCERO C A, FALKINHAM III J O. Health impacts of environmental mycobacteria. Clin Microbiol Rev 2004; 17: 98-106.

2.- VAN INGEN J, BENDIEN S A, DE LANGE W C M, HOEFSLOOT W, DEKHUIJZEN P N R, BOEREE M $\mathrm{J}$, et. al. Clinical relevance of non-tuberculous mycobacteria isolated in the Nijmegen-Arnhem region, The Netherlands. Thorax 2009; 64: 502-6.

3.- VELASCO M, PIFFARDI S, LEPE R, VALENZUELA P. Experiencia de una década en la tipificación y resistencia a fármacos antituberculosos en micobacterias aisladas de pacientes VIH+/SIDA. Rev Chil Enf Respir 2000; 16: 78-84.

4.- ARAYA P, VELASCO M, FERNÁNDEZ J. Identifica- 
ción rápida de micobacterias no tuberculosas mediante análisis de patrones de restricción. Rev Med Chile 2006; 134: 868-73.

5.- RICHTER E, RÜSCH-GERDES S, HILLEMANN D. Evaluation of the GenoType Mycobacterium assay for identification of Mycobacterium species from cultures. J Clin Microbiol 2006; 44: 1769-75.

6.- MORÁN M, ACEVES D, PEÑA P, GALLEGOS M, FLORES S, MONTOYA H, et al. Detección de Mycobacterium tuberculosis mediante la reacción en cadena de la polimerasa en una población seleccionada del noroccidente de México. Rev Panam Salud Pública/Pan Am J Public Health 2000; 7: 389-94.

7.- FRANCO-ÁLVAREZ DE LUNA F, RUIZ P, GUTIÉRREZ J, CASAL M. Evaluation of the GenoType Mycobacteria Direct Assay for detection of Mycobacterium tuberculosis complex and four atypical mycobacterial species in clinical samples. J Clin Microbiol 2006; 44: 3025-7.

8.- $\quad$ GITTI Z, NEONAKIS I, FANTI G, KONTOS F, MARAKI S, TSELENTIS Y. Use of the GenoType Mycobacterium CM and AS to analyze 76 nontuberculous Mycobacterial Isolates from Greece. J Clin Microbiol 2006, 44: 2244-6.

9.- SEAGAR L, PRENDERGAST C, EMMANUEL F, RAYNER A, THOMSON S, LAURENSON F. Evaluation of the GenoType Mycobacteria Direct assay for the simultaneous detection of the Mycobacterium tuberculosis complex and four atypical species in smearpositive respiratory specimens. J Med Microbiol 2008; 57: 605-11.

10.- MÄKINEN J, SARKOLA A, MARJAMÄKI M, VILJANEN M, SOINI H. Evaluation of GenoType and LIPA MYCOBACTERIA assays for identification of finish mycobacterial isolates. J Clin Microbiol 2002; 40: 3478-81.

11.- RUSSO C, TORTOLI E, MENICHELLA D. Evaluation of the new GenoType Mycobacterium assay for identification of Mycobacterial species. J Clin Microbiol 2006; 44: 334-9.

12.- SARKOLA A, MÄKINEN J, MÄRJAMAKI M, MARTTILA H J, VILJANEN M K, SOINI H. Prospective evaluation of the GenoType assay for the routine identification of mycobacteria. Eur J Clin Microbiol Infect Dis 2004; 23: 642-5.

13.- TORTOLI E, PECORARI M, FABIO G, MESSINÒ M, FABIO A. Commercial DNA probes for Mycobacteria incorrectly identify a number of less frecuently encountered species. J Clin Microbiol 2010; 48: 307-10.

14.- TORTOLI E, MARIOTTINI A, MAZZARELLI G. Evaluation of INNO-LiPA Mycobacteria V2: Improved reverse hybridization multiple DNA probe assay for mycobacterial identification. J Clin Microbiol 2003; 41: 4418-20.

Correspondencia a:

Tamara Leiva C.

Sección Micobacterias, Laboratorio Biomédico

Nacional, Instituto de Salud Pública de Chile.

Avda. Marathon $N^{\circ} 1000$, Ñuñoa,

Santiago, Chile.

Fono: 56-2-5755442

E-mail: tleiva@ispch.cl 